\title{
Comparison of protease and aminopeptidase activities in meconium: A pilot study
}

\author{
EWA SKARŻYŃSKA ${ }^{1}$, PAULINA WILCZYŃSKA ${ }^{2}$, BARTOSZ KIERSZTYN $^{3}$, \\ JOANNA ŻYTYŃSKA-DANILUK ${ }^{4}$, ARTUR JAKIMIUK ${ }^{5}$ and BARBARA LISOWSKA-MYJAK ${ }^{1}$ \\ ${ }^{1}$ Department of Biochemistry and Clinical Chemistry, Medical University of Warsaw, Warsaw 02-097; \\ ${ }^{2}$ Department of Biochemistry, Warsaw University of Life Sciences - SGGW, Warsaw 02-776; \\ ${ }^{3}$ Microbial Ecology and Environmental Biotechnology Department, Institute of Botany, Faculty of Biology, \\ University of Warsaw; Biological and Chemical Research Centre, Warsaw 02-089; \\ ${ }^{4}$ Clinical Department of Obstetrics, Female Diseases and Gynaecological Oncology, \\ Central Clinical Hospital of The Ministry of The Interior, Warsaw 02-507; \\ ${ }^{5}$ Reproductive Health Department, Institute of Mother and Child, \\ Warsaw 01-211, Poland
}

Received September 21, 2019; Accepted April 21, 2020

DOI: $10.3892 /$ br.2020.1314

\begin{abstract}
The successive accumulation of proteases and aminopeptidases in meconium are important physiological components of the intrauterine environment in which a fetus develops. The aim of the present study was to assess the changes in the activities of these enzymes in meconium of healthy infants, and to investigate whether there were any statistically significant associations between activity of the enzymes of interest and the mode of delivery. The activities of proteases and aminopeptidases were determined in meconium portions $(n=110)$ using the substrates BODIPY FL casein and L-leucine-7-amido-4-methylcoumarin hydrochloride, respectively. Serial meconium samples (2-5 per neonate) were collected from healthy infants born vaginally $(n=14)$, and by a cesarean section $(n=16)$. Protease activity $\left(10^{4} \mathrm{RFU} / \mathrm{h}\right)$ was lower in the first meconium sample compared with the final sample from the same infant $(3.99 \pm 2.03$ vs. $5.76 \pm 2.24$, respectively, mean \pm standard deviation; $\mathrm{P}=0.004$ ). Conversely, there was no significant difference in aminopeptidase activity $\left(10^{3} \mathrm{nM} / \mathrm{l} / \mathrm{h}\right)$ between consecutive meconium samples $(\mathrm{P}=0.702)$. The ratios of the first-meconium sample enzyme activity to the last-meconium sample enzyme activity were lower for proteases compared with aminopeptidases $(0.76 \pm 0.48$ vs. $1.35 \pm 1.04$, respectively mean \pm standard deviation; $\mathrm{P}=0.014$ ), and sustained in the infants born by a cesarean
\end{abstract}

Correspondence to: Dr Ewa Skarżyńska, Department of Biochemistry and Clinical Chemistry, Medical University of Warsaw, ul. Banacha 1, Warsaw 02-097, Poland

E-mail: ewaskarzynska@wp.pl

Key words: activity, aminopeptidases, proteases, cesarean, vaginal birth, meconium, parturition section $(\mathrm{P}=0.008)$. Spearman's correlation coefficient analysis between the first and last meconium samples showed the correlation increased in the infants born vaginally compared with the rest of the infants (proteases, $\mathrm{R}=0.618$ vs. $\mathrm{R}=0.314$; aminopeptidases, $\mathrm{R}=0.688$ vs. $\mathrm{R}=0.566$ ). Aminopeptidase activity did not exhibit any notable dynamic changes during meconium accumulation in the fetal intestine. In infants born vaginally compared with those born by a cesarean section, the activity of both proteases and aminopeptidases in the first meconium sample showed an improved correlation with the activity of the final meconium sample. This may suggest that in the intrauterine environment, during accumulation of meconium in the digestive tract of the fetus, the activity and/or levels of these enzymes and the substrates they catalyze were more stable in newborns born vaginally compared with infants born by caesarean section.

\section{Introduction}

Proteolytic enzymes (proteases, proteinases and peptidases), belong to Enzyme Classification (EC) number: 3.4. and are a subgroup of hydrolases (EC 3.). Peptidases hydrolyze peptide bonds in fundamentally irreversible reactions (1). They serve a key role in numerous biological processes, including tissue remodeling, wound healing, blood coagulation, angiogenesis, neurogenesis, ovulation, fertilization, hemostasis, immunity, inflammation, senescence, necrosis and apoptosis $(1,2)$. Proteolytic enzymes constitute $\sim 2 \%$ of the mammalian genome and significantly contribute to the enzymatic content and activity of the gastrointestinal tract $(3,4)$. During early pregnancy, secretion of embryo-endometrial proteases is responsible for the hatching of blastocysts and invasion of implanting embryos into the uterine endometrium (5). This physiological uterine modification is largely controlled by proteases and/or their inhibitors, and disorders of the feto-placental homeostasis results in maternal hypertension, 
preterm delivery or preeclampsia (6-10), which are common causes for performing a cesarean delivery.

Aminopeptidases (EC 3.4.11.) are an important subset of proteases which hydrolyze peptide bonds at the N-terminus of proteins and peptides (11). They are generally zinc-dependent metalloenzymes, are responsible for the limited proteolysis and function as posttranslational modifiers of enzymes, inactivators of signal peptides and hormones, and digestive enzymes $(11,12)$.

Meconium is a specific physiological product produced in the gastrointestinal tract of a fetus, obtained noninvasively within 2-4 days after birth and, under normal conditions, is not excreted until/after delivery. Fetal meconium starts forming at 13 weeks of gestation from cycles of swallowed or inhaled amniotic fluid, shed epithelial cells, intestinal secretions and urine (13).

The role of proteolytic enzymes in vital biological processes, including embryogenesis and intrauterine fetal development, may be associated with the mode of delivery. This hypothesis is based on the results of studies which indicate that fecal protease activity is associated with compositional alterations in the intestinal microbiota (4), and that the diversity of the meconium microbiome depends on the mode of delivery $(14,15)$. To date, there have been no studies investigating any possible association between protease activity in the fetal intestine during intrauterine development of the fetus and the mode of delivery, to the best of our knowledge.

The aim of the present study was to assess the changes in the activities of proteases and aminopeptidases, which are a specific pool of proteolytic enzymes present in the meconium of healthy infants, born either vaginally or by a cesarean section, and to investigate whether there are any statistically significant associations between their activity and the mode of delivery.

\section{Materials and methods}

Ethics. The present study was approved by the Medical Ethics Committee at the Central Clinical Hospital of the Ministry of the Interior (Warsaw, Poland) (approval no. 71/2011), and was performed in accordance with the Declaration of Helsinki (16). Written informed consent was obtained from the parents or guardians prior to inclusion of infants in the study.

Profile of neonates. A total of 30 infants born in the Clinical Department of Obstetrics, Female Diseases and Gynecological Oncology, Central Clinical Hospital of the Ministry of the Interior, Warsaw, were included in the present study. Multiple birth neonates, infants born before 35 weeks of gestation or those with a birth weight of $<2 \mathrm{~kg}$ were excluded from the present study. The health conditions of the pregnant women and infants were assessed by the attending physician.

Of the 30 infants, 13 were female and 17 were male; 14 were delivered by vaginal birth and 16 were delivered by cesarean section; the mean gestational age was $38.9 \pm 1.4$ weeks (standard deviation), median gestational age was 39 weeks (range, 36-41 weeks); the mean birth weight was $3,409 \pm 512.5 \mathrm{~g}$, median, 3,445 g (range, 2,260-4,710 g); Apgar scores were (min 1/3/5/10): 10/10/10/10 ( $\mathrm{n}=21)$; 9/10/10/10 ( $\mathrm{n}=1) ; 9 / 9 / 10 / 10$ $(n=1) ; 9 / 9 / 9 / 10(n=2) ; 8 / 9 / 10 / 10(n=5)$. The Apgar score is a test performed on newborns that is repeated every few mins following birth. The test is scored between 0-10, and measures baby's heart rate, breathing, muscle tone, reflex response and appearance.

Sample collection. The meconium was collected in the hospital ward and consisted of all consecutive portions. A total of 110 meconium samples from 30 infants were collected from the nappy with a disposable spatula and transferred into $50 \mathrm{ml}$ graduated plastic containers. The empty containers were weighed prior to adding meconium and reweighed after filling. The date, time and weight of each meconium collection were recorded. Collection was considered complete, when, on inspection, the dark-greenish-black color of the material had changed to the yellowish-brown color characteristic of stool. Passing of meconium stopped between 24-52 h postnatally. Between 2-5 meconium portions were collected from each infant. Analytical-grade distilled water was added to each meconium sample up to a homogenate volume of $45 \mathrm{ml}$ in three stages $(10 \mathrm{ml}, 10 \mathrm{ml}$ and then up to $45 \mathrm{ml})$. Meconium homogenates were stored at $-80^{\circ} \mathrm{C}$. Prior to assessment of protease and aminopeptidase activity, each homogenate sample was thoroughly mixed and individually diluted with analytical-grade distilled water to a final concentration of $0.5 \mathrm{mg}$ meconium per $\mathrm{ml}$ after thawing.

Assay of protease activity. The protease activity in meconium was determined using an EnzChek Protease assay kit (Molecular Probes Inc.). This commercial kit did not involve any separation steps and can be used to measure the kinetics of a variety of exo- and endopeptidases continuously over a wide range of $\mathrm{pH}$ values. The full substrate-dependent kinetic analysis of the meconium protease activity was performed. A set of increasing concentrations of BODIPY FL casein solutions as a substrate was prepared $(2.5,3.75,5.0,7.5,10,20$, 30 and $40 \mu \mathrm{g} / \mathrm{ml})$. Separate stock solutions for each BODIPY FL casein concentration were prepared (a total of 8 different concentrations). A sample with the reaction mixture prepared for the measurement contained $100 \mu 1$ BODIPY FL casein at increasing concentrations and $100 \mu \mathrm{l}$ of individually prepared meconium homogenate solution at a final concentration $0.5 \mathrm{mg} / \mathrm{ml}$. Thus the increasing concentrations of substrates were added into a series of 8 wells and each well contained $100 \mu 1$ meconium homogenate. Samples were assessed in triplicate in a 96-well plate. At time $0 \mathrm{~h}$ and after $1 \mathrm{~h}$ of incubation at room temperature. Fluorescence was measured at $485 \mathrm{~nm}$ (excitation) and $530 \mathrm{~nm}$ (emission) using a Synergy H1 Hybrid Reader (BioTek Instruments, Inc.). The activity of proteases in meconium was calculated as the increase in relative fluorescence units (RFU)/h. For each sample, a mean $\mathrm{RFU} / \mathrm{h}$ was calculated. Autoclaved samples were used as the negative controls.

Assay of aminopeptidase activity. Maximal potential aminopeptidase activity (Vmax) was measured using a fluorogenic substrate (17), according to a modified version of a previously described protocol $(18,19)$. Briefly, $10 \mu \mathrm{l}$ of the substrate L-leucine-7-amido-4-methylcoumarin hydrochloride (Leu-MCA; Sigma-Aldrich; Merck KGaA) solution in ethanol was added to $190 \mu \mathrm{l}$ meconium homogenate (at a 
Table I. Aminopeptidases identified in meconium samples.

Classification system

\begin{tabular}{|c|c|c|c|c|}
\hline UniProtKB & BRENDA & MEROPS & Enzyme name & Abbreviation \\
\hline P28838 & EC 3.4.11.1 & M17.001 & Leucyl aminopeptidase, cytosol aminopeptidase & LAP, cAP \\
\hline P15144 & EC 3.4.11.2 & M01.001 & $\begin{array}{l}\text { Aminopeptidase } \mathrm{N} \text { or } \mathrm{M} \text {, alanine aminopeptidase, } \\
\text { alpha-aminoacyl-peptide hydrolase, alanine or } \\
\text { leucine arylamidase, leucine-beta-naphthylamidase }\end{array}$ & $\begin{array}{l}\text { AP-N, AAP, } \\
\text { CD13 }\end{array}$ \\
\hline Q9UIQ6 & EC 3.4.11.3 & M01.011 & $\begin{array}{l}\text { Placental leucine aminopeptidase, oxytocinase, } \\
\text { cystine aminopeptidase }\end{array}$ & P-LAP, CAP \\
\hline Q9H4A4 & EC 3.4.11.6 & M01.014 & Aminopeptidase B & AP-B \\
\hline Q07075 & EC 3.4.11.7 & M01.003 & $\begin{array}{l}\text { Aminopeptidase A, glutamyl aminopeptidase, } \\
\text { cystyl aminopeptidase }\end{array}$ & AP-A \\
\hline O43895 & EC 3.4.11.9 & M24.005 & Aminopeptidase P, X-Pro aminopeptidase & AP-P \\
\hline
\end{tabular}

Table II. Activity of aminopeptidases stratified by ranges of percentages in meconium samples collected from 30 neonates.

\begin{tabular}{lccccc}
\hline & & \multicolumn{2}{c}{ Aminopeptidase activity $\left(10^{3} \mathrm{nM} / \mathrm{l} / \mathrm{h}\right)$} & \\
\cline { 3 - 5 } Percentage & $\mathrm{n}$ & Mean \pm standard deviation & Median & Range & $\begin{array}{c}\text { Kruskal-Wallis-ANOVA } \\
\text { P-value }\end{array}$ \\
\hline $0-25 \%$ & 29 & $1.27 \pm 0.99$ & 0.98 & $0.10-4.31$ & \\
$26-50 \%$ & 23 & $1.08 \pm 0.68$ & 1.13 & $0.01-3.01$ & $\mathrm{P}=0.702$ \\
$51-75 \%$ & 21 & $1.87 \pm 1.79$ & 1.10 & $0.05-6.64$ & \\
$76-100 \%$ & 37 & $1.31 \pm 1.15$ & 1.02 & $0.11-5.29$ & \\
\hline
\end{tabular}

concentration of $0.5 \mathrm{mg}$ meconium $/ \mathrm{ml}$ ), resulting in solutions with final Leu-MCA concentrations of 1.0, 2.5, 5.0, $10,15,20,25$ or $30 \mu \mathrm{M}$. Separate stock solutions for each Leu-MCA concentration were prepared (a total of 8 concentrations). For preparation of the calibration standard solution, 7-amino-4-methylcoumarin (MCA) was used to measure the fluorescence. Final MCA concentrations in ethanol were $4.688,9.375,18.75,37.5,75,150,300$ or $600 \mathrm{nM}$. A calibration curve was prepared individually for each meconium homogenate. Samples were loaded in triplicate into a 96-well plate. At time $0 \mathrm{~h}$ and after $1 \mathrm{~h}$ of incubation at room temperature, the fluorescence of the MCA product was determined spectrofluorometrically at $380 \mathrm{~nm}$ (excitation) and $460 \mathrm{~nm}$ (emission) using a Synergy H1 Hybrid Reader (BioTek Instruments, Inc.). The aminopeptidase activity calculated from the standard curve is given in $\mathrm{nM} / \mathrm{l} / \mathrm{h}$.

Data analysis. Both methods of enzyme activity measurement used the enzymatic hydrolysis of substrates and detection of the fluorescence of the products. The tested enzyme-substrate system followed first-order Michaelis-Menten kinetics (20). Plotting reaction velocity (v) against substrate concentration [S] resulted in a rectangular hyperbola-shaped relationship, described by the equation: $\mathrm{v}=\mathrm{V}_{\max } \mathrm{x}[\mathrm{S}] /\left(\mathrm{K}_{\mathrm{m}}+[\mathrm{S}]\right)$, where $\mathrm{K}_{\mathrm{m}}$ is the Michaelis constant and $\mathrm{V}_{\max }$ is the maximal potential activity. $\mathrm{K}_{\mathrm{m}}$ represents the concentration of substrate which permits the enzyme to achieve half $\mathrm{V}_{\max }$.
Plots of reaction velocity against substrate concentration for proteases and aminopeptidases were constructed and $\mathrm{V}_{\max }$ and $\mathrm{K}_{\mathrm{m}}$ were calculated. Non-linear regression analysis was used to calculate the kinetic parameters of enzymatic reactions using Origin 6.1 (OriginLab Corporation).

Aminopeptidases identified in the meconium samples (21) are listed in Table I according to their classification system in the following online databases; uniprot.org, brenda-enzymes. org and merops.sanger.ac.uk.

Statistical analysis. Statistical analysis was performed using Statistica Version 12 (StatSoft Inc.). The results are presented as the mean \pm standard deviation, or median and range. A Shapiro-Wilk's test was performed to assess the normality of distribution. A Mann-Whitney U-test and a Kruskal-Wallis with a Fisher's LSD post hoc test or an ANOVA with a post-hoc Tukey's test, were used to compare the activity of peptidases and aminopeptidases. A Spearman's rank-order correlation test was performed to determine the relationship between the activity of proteases and aminopeptidases in the first and last meconium portions. $\mathrm{P}<0.05$ was considered to indicate a statistically significant difference.

\section{Results}

Table II shows the activity of aminopeptidases $\left(10^{3} \mathrm{nM} / \mathrm{l} / \mathrm{h}\right)$ in successive percentage ranges of meconium weight. It was 
Table III. Ratio of protease and aminopeptidase activities in the first and last meconium.

Protease activity $\left(10^{4} \mathrm{RFU} / \mathrm{h}\right)$

Aminopeptidase activity $\left(10^{3} \mathrm{nM} / \mathrm{l} / \mathrm{h}\right)$

\begin{tabular}{|c|c|c|c|c|c|c|}
\hline \multirow[b]{2}{*}{ Meconium } & & & & & & \\
\hline & Mean \pm SD & Median & Range & Mean \pm SD & Median & Range \\
\hline First & $3.99 \pm 2.03$ & 3.79 & $0.35-8.34$ & $1.24 \pm 0.94$ & 1.04 & $0.09-4.31$ \\
\hline Last & $5.76 \pm 2.24$ & 5.92 & $0.98-10.66$ & $1.29 \pm 1.22$ & 0.91 & $0.11-5.29$ \\
\hline
\end{tabular}

Ratio for protease

Ratio for aminopeptidase

\begin{tabular}{|c|c|c|c|c|c|c|}
\hline Ratio & Mean \pm SD & Median & Range & Mean \pm SD & Median & Range \\
\hline First/last & $0.76 \pm 0.48$ & 0.77 & $0.08-2.68$ & $1.35 \pm 1.04$ & 0.99 & $0.15-4.11$ \\
\hline
\end{tabular}

$\mathrm{SD}$, standard deviation; RFU, relative fluorescence units.

assumed that the weight of meconium excreted after birth varied among neonates, but was always equal to $100 \%$ of the weight of meconium formed and accumulated in the intestine during gestation. Total meconium weight per neonate ranged from 0.579-32.086 g (mean, 10.92 \pm 8.1 ; median, 9.19). Each subsequent portion of meconium collected from an individual neonate represented the specific activity of an aminopeptidase and a specific range (\%) of the total meconium weight. Subsequently, for each of the calculated percentage ranges, the value corresponding to $50 \%$ of this range was calculated. This value was assigned to one of the four percent ranges: $0-25$, 26-50, 51-75 and 76-100\%. The number of aminopeptidases activity measurements in percent ranges varied as the number of meconium portions excreted, and the total weight of meconium accumulated in the fetal intestine were neonate-specific and differed among infants. Thus, aminopeptidase activities in the $0-25$ and $76-100 \%$ ranges did not invariably correspond to the measurements in the first and last meconium.

The median values were closer than the mean values in sequential percent ranges and there were no statistically significant differences in the aminopeptidase activity values demonstrated within these ranges $(\mathrm{P}=0.702)$.

Table III shows the activity of proteases and aminopeptidases in the first and last meconium portions, and the ratio of enzyme activity of first to last meconium portion. Fig. 1 shows the distribution of the measurements in individual infants, including the mode of delivery.

A statistically significant increase in the activity of proteases was demonstrated between the first and last meconium sample $(\mathrm{P}=0.004)$, and between the meconium the last portions from the neonates born vaginally and those delivered by a cesarean section $(\mathrm{P}=0.038)$. The activities of both proteases (meconium from 24 neonates) and aminopeptidases (meconium from 15 neonates) were lower in the first meconium portion compared with the last meconium portion. In 5 neonates, the activity of proteases in the first meconium portion was slightly higher compared with the last meconium portion and the ratios did not exceed 1.25 , except in only one neonate (born by a cesarean section) where the ratio was 2.68. Unlike protease activity, the activity of aminopeptidases was significantly higher in the first meconium portion compared with the last meconium portion in 8 neonates, and the ratio exceeded 2 , the maximum ratio observed was 4.11 in a neonate born vaginally. Statistically significant differences were observed in the first to last meconium portion ratio for proteases and aminopeptidases in the entire study group $(\mathrm{P}=0.014)$ and in neonates born by cesarean birth $(\mathrm{P}=0.008)$.

Fig. 2 shows the association between the activity of proteases and of aminopeptidases in the first and last meconium portion, including the mode of delivery. Vaginal birth was associated with higher Spearman's correlation coefficients values relative to the entire study group for both proteases ( $\mathrm{R}=0.618$ and $\mathrm{R}=0.314$, respectively) and aminopeptidases ( $\mathrm{R}=0.688, \mathrm{R}=0.566$, respectively).

\section{Discussion}

The assessment of protease and aminopeptidase activity in the meconium of healthy infants, and the interrelationship of these enzymes was demonstrated for the first time in the present study, to the best of our knowledge. The results showed that during fetal intrauterine development, activity of these enzymes may be associated with the mode of delivery. Increases in the protease activity between the first and last meconium portions, observed in $80 \%$ of the infants included in the study, confirmed the results of our previous (22). Peptidases are a highly diverse class of enzymes and the MEROPS database is an essential source of information regarding peptidases, their substrates and inhibitors (23). In total, 22 human aminopeptidases are described in the MEROPS database and 6 of these were present in meconium (Table I) (21). In addition, proteomic analysis has shown a total of 265 enzymatic proteins in meconium, $25 \%$ of which are proteases, and of these, $\sim 9 \%$ are aminopeptidases (data not shown). The activities of aminopeptidases assayed in the present study represent a pool of six enzymes with important roles in fetal development, such as the regulation of pressure, neuronal cell function in the brain, learning and memory, or final digestion of peptides $(8,11)$. Meconium aminopeptidases are identical to placental aminopeptidases (8), but an additional leucyl aminopeptidase (EC 3.4.11.1) is present in meconium which may be derived from activated lymphocytes (24). As the activities of aminopeptidases showed no apparent changes 

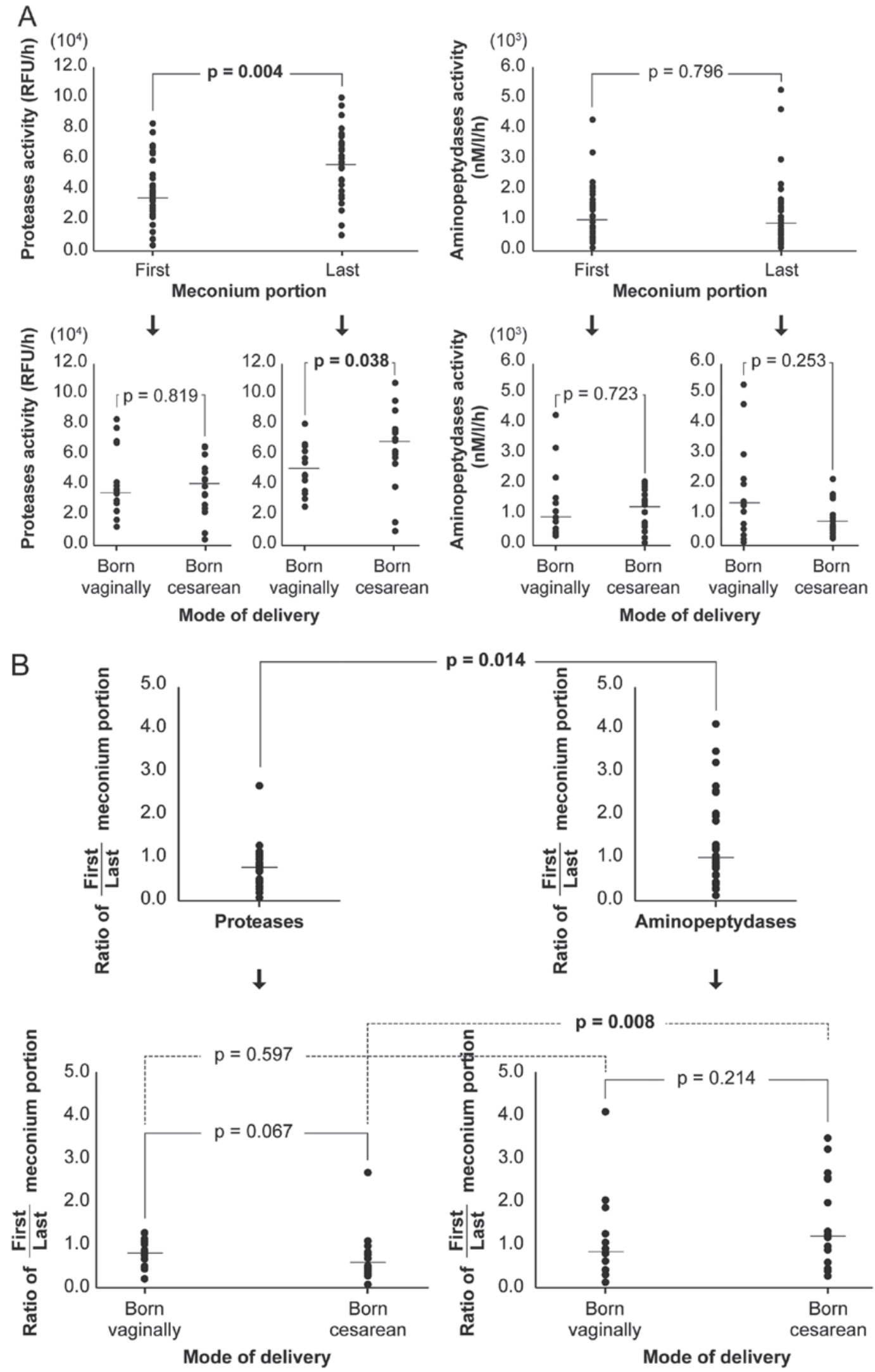

Figure 1. Scatter plots of enzyme activity in meconium. (A) Protease and aminopeptidase activities in the first and last meconium samples, and (B) the ratios of enzyme activity between the first and last samples. Data from 30 infants, including the mode of delivery. Vertically arranged dots represent individual protease and aminopeptidase activity measurements. The horizontal lines represent median levels. RFU, relative fluorescence units.

between successively collected portions of meconium, this may suggest a similar degree of their involvement throughout gestation. However, as a pool of aminopeptidases was assayed, the actual involvement of individual enzymes may vary at different stages of gestation. To date, the activity of these aminopeptidases has not been investigated in meconium. 

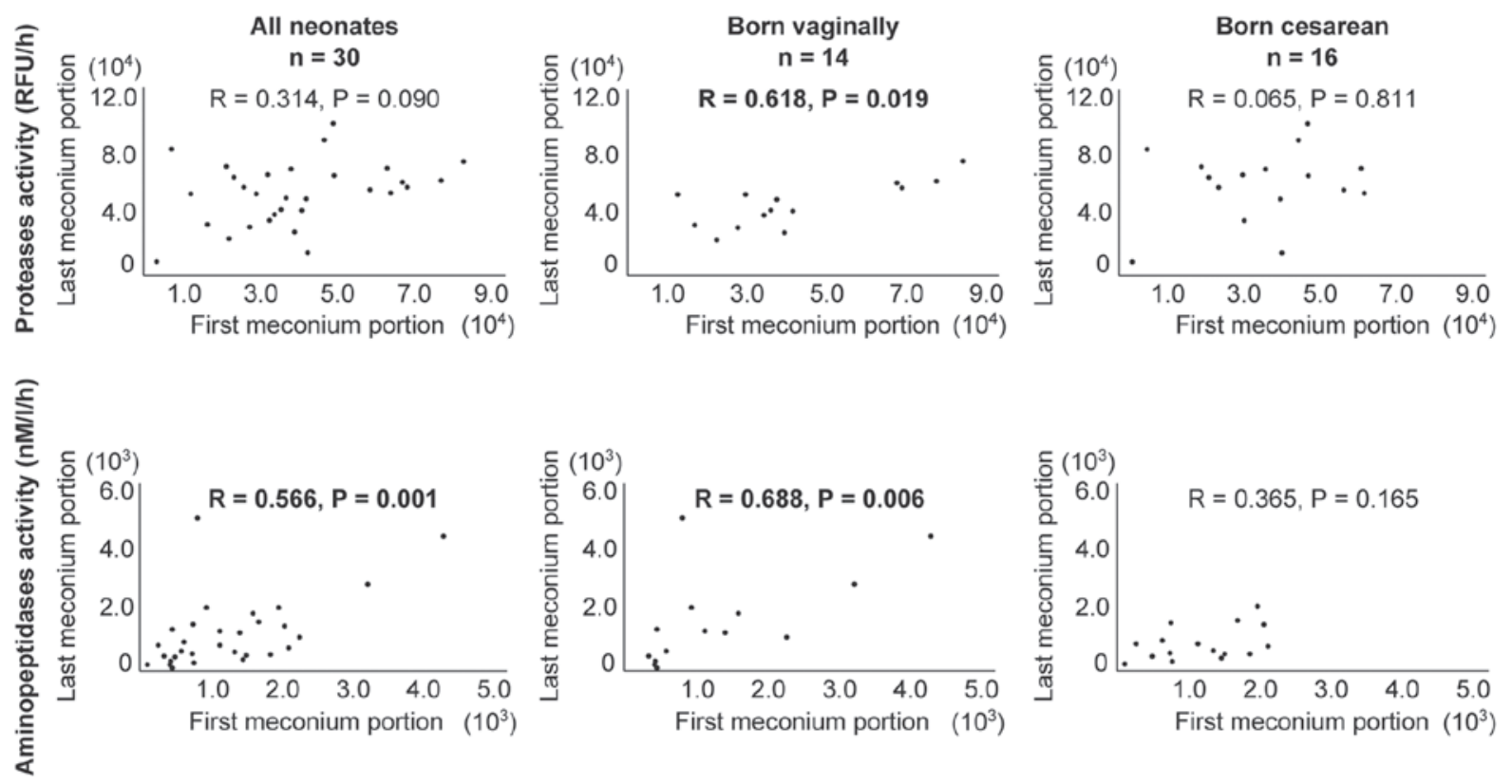

Figure 2. Statistically significant Spearman correlation coefficients of the protease and aminopeptidase activity between the first and last meconium sample, including the mode of delivery. R, Spearman correlation coefficient; RFU, relative fluorescence units.

Placental leucine aminopeptidase (P-LAP) appears to be the most frequently studied aminopeptidase $(6-8,11,25-27)$. In the serum of normal pregnant women, P-LAP shows a characteristic pattern of activity. P-LAP activities in the serum of normal pregnant women are constant during the early stages of pregnancy, then rise between the 18th and 37th weeks, peaking at 38 weeks of gestation. The activities are flat or slightly decreased just before labor and then further decrease within 4 weeks after delivery (25). The possible sources of serum P-LAP include the placenta, umbilical vessels, decidua and/or the fetus (25). However, whether meconium may be an additional source of serum P-LAP, or if serum P-LAP is present in the meconium has not been determined, to the best of our knowledge. High expression levels of P-LAP have been reported in the lung, kidney, heart and pancreas (26), and it is involved in hormonal control of uterine contraction, osmoregulation and blood glucose level regulation (27). Aminopeptidase $\mathrm{N}$ serves a role in signal transduction, inflammation, immunological responses, matrix degradation, chemokines and cytokines processing (28). Aminopeptidases are an important subgroup of peptidases which serve a key role in a variety of processes, such as regulation of hormone levels, protein maturation, inactivation of proteins and protein digestion (25-29). Further research is required to determine the activity of each of the aminopeptidases identified in meconium in the present study, and to measure their activities in the maternal serum and/or amniotic fluid. Individual assessment of specific aminopeptidases and other proteases is essentially hampered by the need for specific substrates or selective inhibitors. Research is currently being performed in this area, particularly for aminopeptidase N (28), and another study used artificial substrates for aminopeptidase A, aminopeptidase B and aminopeptidase $\mathrm{N}$ (29).

The source of proteases and aminopeptidases in meconium has not been definitively established, to the best of our knowledge. They are hypothesized to be derived from the mother, placenta, amniotic fluid and/or fetus, but other studies have also demonstrated aminopeptidase activity is associated with bacteria $(4,30)$. The paradigm regarding the sterile fetal environment is now being challenged based on evidence showing the presence microbial communities in meconium (31). A previous study demonstrated that the diversity of the meconium microbiome was higher in vaginally delivered infants compared with infants born by cesarean section, with the Propionibacterium species being the most abundant in the former and Bacillus licheniformis in the latter (14). Another study found significant associations between specific intestinal bacterial groups and fecal protease activity with a significant difference between the microbiotas of high vs. low fecal protease activity samples, suggesting that the difference in microbial communities between these groups is based on both dominant and low abundance bacterial taxa present in fecal samples (4). However, the meconium microbiome may also be dependent on race or geographical differences as well as the interval of meconium collection (14).

The health condition of all neonates included in the present study was assessed and considered good by the attending physician. There was a stronger association between the activities of proteases and aminopeptidases in the first vs. the last meconium in vaginally born infants compared with the entire study group, which may suggest in utero homeostasis of processes catalyzed by these enzymes, while in infants born by a cesarean section, this homeostasis may have been disturbed. In the present study, 10 of the 16 infants born by a cesarean section were born by an emergency cesarean section (fetal asphyxia or failure to progress), while 6 infants were born by an elective cesarean section. Considering the rising rates of cesarean sections, both elective and emergency (32), additional studies are required to characterize and differentiate protease and individual aminopeptidase activities associated with the mode of delivery, particularly for infants born by cesarean section. It may also be important to consider the 
pharmacological aspects when analyzing individual aminopeptidases.

In summary, in the present study, it was demonstrated that there was an absence of significant dynamic changes in the aminopeptidase activity during meconium accumulation in the fetal intestine. In infants born vaginally, compared with those born by a cesarean section, there was a stronger association between the activities of proteases and aminopeptidases in the first vs. the last meconium portion, which may suggest a more prominent homeostatic effect of processes catalyzed by these enzymes in the fetal intestine or even in the intrauterine environment. These results, however, should be confirmed in a larger number of infants, taking into account the type of cesarean section (elective vs. emergency) and whether this affects the activity of enzymes. Additionally, the activity of individual aminopeptidases identified in meconium should be determined.

\section{Acknowledgements}

The authors would like to thank Dr Donat Jaguś at The Clinical Department of Obstetrics, Female Diseases and Gynecological Oncology, Central Clinical Hospital of the Ministry of the Interior in Warsaw for systematic documentation and valuable medical tips.

\section{Funding}

This work was supported by a Research Project Funded by The National Center for Science (approval no. DEC-2011/01/B/ NZ7/00648).

\section{Availability of data and materials}

The datasets used and/or analyzed during this study are available from the corresponding author upon reasonable request.

\section{Authors' contributions}

ES and BL-M conceived and designed the study and drafted the initial manuscript. ES analyzed the data. PW collected materials, and measured and analyzed the data. BK measured and analyzed the data. JZ̈-D and AJ provided medical supervision of meconium collection and medical documentation. All authors have read and approved the final version of the manuscript.

\section{Ethics approval and consent to participate}

This study was approved by the Medical Ethics Committee (approval no. 71/2011) at the Central Clinical Hospital of the Ministry of the Interior (Warsaw, Poland), in accordance with the Declaration of Helsinki. Written informed consent was obtained from the parents prior to inclusion of infants in the study.

\section{Patient consent for publication}

Not applicable.

\section{Competing interests}

The authors declare that they have no competing interests.

\section{References}

1. Pérez-Silva JG, Español Y, Velasco G and Quesada V: The Degradome database: Expanding roles of mammalian proteases in life and disease. Nucleic Acids Res 44: D351-D355, 2016

2. López-Otín C and Bond JS: Proteases: Multifunctional enzymes in life and disease. J Biol Chem 283: 30433-30437, 2008.

3. Antalis TM, Shea-Donohue T, Vogel SN, Sears C and Fasano A: Mechanisms of disease: Protease functions in intestinal mucosal pathobiology. Nat Clin Pract Gastroenterol Hepatol 4: 393-402, 2007.

4. Carroll IM, Ringel-Kulka T, Ferrier L, Wu MC, Siddle JP, Bueno L and Ringel Y: Fecal protease activity is associated with compositional alterations in the intestinal microbiota. PLoS One 8: e78017, 2013

5. Seshagiri PB, Lalitha HS, Mishra A and Sireesha GV: Embryo-endometrial proteases during early mammalian development. Indian J Exp Biol 41: 756-763, 2003.

6. Mizutani S and Tomoda Y: Effects of placental proteases on maternal and fetal blood pressure in normal pregnancy and preeclampsia. Am J Hypertens 9: 591-597, 1996.

7. Mizutani S, Wright J and Kobayashi H: A new approach regarding the treatment of preeclampsia and preterm labor. Life Sci 88: 17-23, 2011.

8. Mizutani S: Physiological roles of placental proteases in feto-placental homeostasis. Nagoya J Med Sci 61: 85-95, 1998.

9. Townsend R, O'Brien P and Khalil A: Current best practice in the management of hypertensive disorders in pregnancy. Integr Blood Press Control 9: 79-94, 2016.

10. Kattah AG and Garovic VD: The management of hypertension in pregnancy. Adv Chronic Kidney Dis 20: 229-239, 2013.

11. Sanderink GJ, Artur Y and Siest G: Human aminopeptidases: A review of the literature. J Clin Chem Clin Biochem 26: 795-807, 1988.

12. Byzia A, Szeffler A, Kalinowski L and Drag M: Activity profiling of aminopeptidases in cell lysates using a fluorogenic substrate library. Biochimie 122: 31-37, 2016.

13. Park BY and Lee BK: Use of meconium in perinatal epidemiology: Potential benefits and pitfalls. Ann Epidemiol 24: 878-881, 2014.

14. Shi YC, Guo H, Chen J, Sun G, Ren RR, Guo MZ, Peng LH and Yang YS: Initial meconium microbiome in Chinese neonates delivered naturally or cesarean section. Sci Rep 8: 3255, 2018.

15. Nagpal R, Tsuji H, Takahashi T, Kawashima K, Nagata S, Nomoto K and Yamashiro Y: Sensitive quantitative analysis of the meconium bacterial microbiota in healthy term infants born vaginally or by cesarean section. Front Microbiol 7: 1997, 2016.

16. World Medical Association: World medical association declaration of Helsinki: Ethical principles for medical research involving human subjects. JAMA 310: 2191-2194, 2013.

17. Hoppe HG: Significance of exoenzymatic activities in the ecology of brackish water: Measurements by means of methylumbelliferyl-substrates. Mar Ecol Prog Ser 11: 299-308, 1983.

18. Kiersztyn B, Siuda W and Chróst RJ: Persistence of bacterial proteolytic enzymes in lake ecosystems. FEMS Microbiol Ecol 80: 124-134, 2012.

19. Chróst RJ: Microbial ectoenzymes in aquatic environments. In: Overbeck J \& Chróst RJ, eds. Aquatic microbial ecology: Biochemical and molecular approaches. Springer-Verlag: New York, 47-78, 1990.

20. Johnson KA and Goody RS: The original Michaelis constant: Translation of the 1913 Michaelis-Menten paper. Biochemistry 50: 8264-8269, 2011

21. Lisowska-Myjak B, Skarżyńska E, Wojdan K and Nasierowska-Guttmejer A: Protein and peptide profiles in neonatal meconium. J Obstet Gynaecol Res 45: 556-564, 2019.

22. Skarżyńska E, Kiersztyn B, Wilczyńska P, Jakimiuk A and Lisowska-Myjak B: Total proteolytic activity and concentration of alpha-1 antitrypsin in meconium for assessment of the protease/antiprotease balance. Eur J Obstet Gynecol Reprod Biol 223: 133-138, 2018

23. Rawlings ND, Barrett AJ, Thomas PD, Huang X, Bateman A and Finn RD: The MEROPS database of proteolytic enzymes, their substrates and inhibitors in 2017 and a comparison with peptidases in the PANTHER database. Nucleic Acids Res 46: D624-D632, 2018. 
24. Sugaya N, Takeuchi Y and Kanno T: Increased cytosol aminopeptidase in serum in measles infection. Clin Chem 34: 212-213, 1988.

25. Yamahara N, Nomura S, Suzuki T, Itakura A, Ito M, Okamoto M, Tsujimoto M, Nakazato M and Mizutani S: Placental leucine aminopeptidase/oxytocinase in maternal serum and placenta during normal pregnancy. Life Sci 66: 1401-1410, 2000.

26. Kobayashi H, Nomura S, Mitsui T, Ito T, Kuno N, Ohno Y, Kadomatsu K, Muramatsu K, Nagasaka T and Mizutani S: Tissue distribution of placental leucine aminopeptidase/oxytocinase during mouse pregnancy. J Histochem Cytochem 52: 113-121, 2004.

27. Matsumoto $\mathrm{H}$ and Mori T: Changes in cystine aminopeptidase (oxytocinase) activity in mouse serum, placenta, uterus and liver during pregnancy or after steroid hormone treatments. Zoolog Sci 15: 111-115, 1998.
28. Grzywa R, Oleksyszyn J, Salvesen GS and Drag M: Identification of very potent inhibitor of human aminopeptidase N (CD13). Bioorg Med Chem Lett 20: 2497-2499, 2010.

29. Gard PR, Fidalgo S, Lotter I, Richardson C, Farina N, Rusted J and Tabet N: Changes of renin-angiotensin system-related aminopeptidases in early stage Alzheimer's disease. Exp Gerontol 89: 1-7, 2017.

30. Gonzales T and Robert-Baudouy J: Bacterial aminopeptidases: Properties and functions. FEMS Microbiol Rev 18: 319-344, 1996.

31. Neu J and Rushing J: Cesarean versus vaginal delivery: Long-term infant outcomes and the hygiene hypothesis. Clin Perinatol 38: 321-331, 2011.

32. Arboleya S, Suárez M, Fernández N, Mantecón L, Solís G, Gueimonde M, de Los Reyes and Gavilán CG: C-section and the neonatal gut microbiome acquisition: Consequences for future health. Ann Nutr Metab 73 (Suppl 3): 17-23, 2018. 